\title{
LA GRAMÁTICA INTEGRATIVA
}

\author{
Ángela Gracia Menéndez
}

En el presente trabajo pretendemos exponer los fundamentos de la Gramática Integrativa, desarrollada por Hans-Heinrich Lieb y Peter Eisenberg en Berlin. ${ }^{1}$

Las bases de este enfoque suponen un replanteamiento de las exigencias a una teoría gramatical, que durante decenios se identificó con la Gramática Generativa. Desde esa fecha no hubo ningún enfoque que causara mayor impacto dentro de la teoría gramatical.

En Europa, no obstante, la discusión dentro del campo sintáctico fue dominada por la teoría de dependencia, que posteriormente pasó a denominarse teoría de valencias. Dicha teoría elaborada por Lucien Tesnière en "Esquisses d'une Syntaxe Structurale"2 y perfeccionada en Éléments de Syntaxe Structurale. ${ }^{3}$ parte de la idea de que el número y tipo de complementos de un verbo depende de los lugares vacíos que abre éste.

Este enfoque ha tenido gran repercusión en la teoría gramatical desarrollada en Alemania, pero, en un sentido estricto, no puede considerarse una 'gramática', o sea, una teoría sobre un sistema, como puede ser el alemán, el inglés o el español, dado que se limita a una descripción de la relación de subordinación y a la explicación de la translación. Obviamente, el sistema de una lengua es más complejo y no puede reducirse a dichas relaciones.

Uno de los objetivos dentro de este enfoque es explicar la composición del contenido semántico a través de las palabras que son enunciadas, ya que la finalidad de toda comunicación es transmitir mensajes, y para ello se recurre a palabras que son enunciadas en un determinado orden. Para ello habrá que tener especial consideración no sólo del contenido semántico de todas las palabras sino también del significado de las funciones sintácticas que desempeñan éstas. La ventaja de este enfoque es indudablemente que fue concebido de manera que pueda ser aplicable a todas las lenguas.

La sintaxis integrativa se caracteriza por ser una sintaxis de superficie que sirve de base para una semántica. Esto significa que a las estructuras sintácticas les es asignado un

\footnotetext{
${ }^{1}$ Véase P. Eisenberg, Oberflächenstruktur und logische Struktur. (Tübingen 1976).

P. Eisenberg, "Integrative Syntax." Lieb (ed.): Oberflächensyntax und Semantik. (Tübingen 1980)

H.-H. Lieb, Integrational Linguistics. General Outline. Vol. 1. (Amsterdam 1983)

H.-H. Lieb, "Integrative Semantik." Lieb (ed.): Oberflächensyntax und Semantik. (Tübingen 1980)

${ }^{2}$ L. Tesnière, Esquisses d'une Syntaxe Structurale (Paris 1959)

3 . L. Tesnière, Éléments de Syntaxe Structurale. (Paris 1980 ${ }^{4}$ )
} 
contenido semántico. El significado, que es asignado basándose en la estructura sintáctica, es concebido como una función del significado de unidades sintácticas. ${ }^{4}$

La limitación a la estructura de superficie tiene como consecuencia, que sólo son descritas aquellas unidades correspondientes a palabras fonológicas, excluyendo por tanto todo tipo de abstracciones creadas desde un punto de vista lógico o semántico. ${ }^{5}$

O sea, que no se admiten categorías abstractas para la composición del significado, sino únicamente las palabras, que efectivamente son enunciadas, aparecen en la superficie.

Para poder asignar un significado a una frase se asume que las unidades sintácticas han de ser fijadas únicamente con los siguientes medios sintácticos del sistema de idiolectos. (Lieb 1983:53):

1. El orden de las formas sintácticas:

La cadena hablada es una secuencia de palabras. El orden en el que aparecen las palabras, determina en gran medida el significado de una frase.

2. La marcación morfológica de las unidades sintácticas:

La estructura de marcación consta de dos partes:

a) Las categorías del paradigma ("Paradigmenkategorien") que incluyen información que atañe al paradigma entero.

Para un verbo incluye información como clase de verbo ('VOLLVERB' o 'KOPULAVERB'). Sin embargo, para un sustantivo comprende información como GENERO.

b) Las categorías de la unidad ("Einheitenkategorien")

Aquí se describe la unidad sintáctica tal como aparece en la frase en cuestión. Para un verbo se indica la persona, número, tiempo, modo y voz. Para un sustantivo, en cambio, se señala el caso y el número.

3. La entonación

La entonación de un enunciado puede ser de gran importancia, siempre que el enunciado sea ambiguo desde un punto de vista estructural.

Así, p. ej., el enunciado Die Mutter liebt die Tochter es ambiguo. Con una entonación no marcada puede significar que la madre ama a la hija, pero si acentuamos la palabra Mutter adquiere la siguiente lectura: La hija ama a la madre (y no al padre). Esta ambigüedad sólo se produce porque el nominativo y el acusativo del sustantivo femenino siempre es die. ${ }^{6}$

\footnotetext{
${ }^{4}$ H.-H. Lieb, Integrational Linguistics. General Outline Vol. 1. (Amsterdam 1983) 52 - 53.

5 P. Eisenberg/ D. Hartmann/ G. Klann/ H.-H. Lieb, Oberflächensyntax und syntaktische Konstituentenstrukturen des Deutschen. FU-Berlin Linguistische Arbeiten und Berichte 4 (1975). 66

${ }^{6}$ Véase P. Eisenberg/ D. Hartmann/ G. Klann/ H.-H. Lieb (1975) 100 -101
} 
Además del significado de las estructuras sintácticas se le atribuye un papel considerable a las relaciones gramaticales, a las que también se les asigna un contenido semántico, que garantizan la composición del significado de la frase de la suma del significado de las unidades sintácticas. (Lieb 1983:66)

Las relaciones gramaticales tradicionales son formalmente concebidas como funciones cuyos argumentos son cuadruples ( $\mathrm{f}, \mathrm{s}, \mathrm{e}, \mathrm{S}$ ), entre una unidad sintáctica o un encadenamiento $\mathrm{f}$, la estructura sintáctica $\mathrm{s}$, la interpretación léxica e y el sistema de idiolectos S. Los valores de la función son concebidos como relaciones de dos términos.

Debido a que el significado de las funciones gramaticales se aplica en el proceso de composición simultáneamente en una frase y que el contenido semántico no se puede asignar por separado a las distintas funciones gramaticales, Lieb abandona la asunción tradicional de distintas funciones gramaticales en favor de una función de complemento de ntérminos, según la valencia del predicado de la frase.

$\mathrm{El}$ inconveniente que puede tener hablar sólo de 'complementos de n-términos' es que las diferentes funciones gramaticales como es 'sujeto', 'objeto de dativo' u 'objeto de acusativo' quedan algo oscurecidas. Una diferencia relevante para la composición del significado de la frase radica precisamente en que un constituyente tiene la función de sujeto o bien de objeto. Los términos equivalentes propuestos por Lieb, como 'complemento 1' o 'complemento 2' quedan demasiado abstractos, por lo que habría que dar explicaciones adicionales en las que éstas tendrían que ser equiparadas a un dativo o bien a un acusativo. En definitiva, es un procedimiento superfluo que no constituye una aportación significativa.

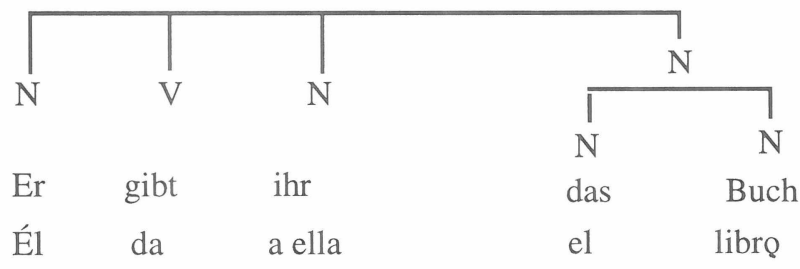

En la frase ( 1 ) los términos 'comp ${ }^{1}$ ', 'comp ${ }^{21}$ y 'comp 3 ' no especifican para nada la estructura de la frase, y que, en concreto, se trata de un sujeto, un objeto en acusativo y otro en dativo.

Esta innovación de Lieb, en todo caso, no ha tenido mayor repercusión en esa escuela, prueba de ello es que el Grundriß der deutschen Sprache de Peter Eisenberg (1986), que puede considerarse la primera aplicación de esa teoría gramatical al sistema del alemán, no retoma la propuesta de Lieb, sino que Eisenberg sigue hablando de las relaciones gramaticales tradicionales.

Uno de los fundamentos de este enfoque gramatical es el de partir de la teoría de valencias. 
Tesnière concibe el verbo como regente del que dependen los actantes. Proyectado en un estema, el verbo es una 'palabra superior' y el actante una 'palabra inferior', que siempre es un sustantivo, lo que es visualizado en un estema gráficamente situando el verbo en el centro, mientras que los complementos se hallan en un plano inferior (véase frase (2)).

Tesnière no explicita dichos términos, que dentro de su concepción son relevantes. No obstante, la equiparación 'verbo - núcleo' y 'actante - sustantivo' nos permite asumir que Tesnière tenía una concepción de valencia meramente sintáctica.

En la Gramática Integrativa, los diagramas utilizados son diferentes; han sido adaptados a las exigencias de una gramática de superficie manteniendo un plano lineal. El predicado de la frase no aparece a un nivel más alto sino que el verbo está coordinado a sus complementos.

Las constituyentes que tienen función de complemento han de ser averiguados a través de la estructura de marcación. En las categorías del paradigma, que per definitionem se señalan siempre con mayúscula, se establece la categoría de régimen del predicado y posteriormente se localiza el constituyente con la categoría correspondiente dentro de la categoría de unidad.

Como ya mencionamos, este enfoque es una gramática de superficie que reconstruye el significado de la frase a través del significado de la suma de las palabras que constituyen el enunciado. Precisamente el medio sintáctico más elemental el es orden de las formas sintácticas.

El estema de Tesnière señala la jerarquía, pero, en un sentido estricto, no visualiza la estructura de la frase en cuestión, dado que no entra en cuestiones de relaciones gramaticales entre los constituyentes de la frase.

Además, hay estemas tan complicados que casi no permiten la reconstrucción exacta de la frase, excepto tal vez para un hablante nativo. Puede incluso darse el caso de que el orden de determinados sintagmas varíe dentro de la gramaticalidad. Partiendo de algunos estemas propuestos por Tesnière difícilmente se lograría la reconstrucción de tales frases.

(2)

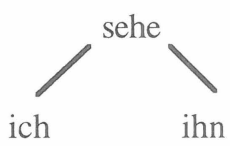

(3)

$\begin{array}{lll}\mathrm{N} & \mathrm{V} & \mathrm{N} \\ \text { Ich } & \text { sehe } & \text { ihn }\end{array}$

En la Gramática Integrativa, en cambio, la secuencia del enunciado es primordial, permanece inalterada. El diagrama refleja la estructura de la frase, pero partiendo siempre de la secuencia dada, sin modificación alguna. La frase es concebida como abstracción; es la representación escrita de un enunciado, y éste, a la vez, es una secuencia de palabras ("Wortfolge $\mathrm{f}^{\prime}$ ), que también puede constar de un elemento.

Es natural, que puedan surgir dificultades en gramáticas de superficie, a la hora de describir lenguas como el alemán, dado que las formas verbales perifrásticas pueden ser 
discontínuas. Pueden hasta incluir el objeto de dativo o de acusativo entre el verbo auxiliar y el participio perfecto, al contrario que en español.

(4)

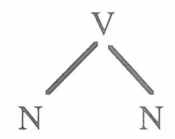

Ich habe ihn gesehen

(Yo he lo visto)

(5)

$\begin{array}{llll}\text { Sie } & \text { hat } & \text { ihm } & \text { vergeben } \\ \text { Ella } & \text { ha } & \text { le } & \text { perdonado }\end{array}$

En lo que sigue, haremos un análisis de la frase ( 4 ), con el fin de esbozar los fundamentos de la Gramática Integrativa.

La forma habe gesehen es la $1^{\text {a }}$ persona, singular del indicativo perfecto, activo del paradigma verbal sehen ${ }^{\mathrm{P} 6}$. Habe sólo tiene una función sintáctica, como es la de marcar el modo, persona, número, tiempo y voz, mientras que gesehen recoge el contenido léxico, que es idéntico para el paradigma entero.

Dado que no se puede asignar una categoría sintáctica a las dos formas aisladamente, por la razón arriba expuesta, se procede a unirlas mediante una línea horizontal, y, posteriormente, se les asigna la categoría 'verbo', puesto que es un elemento del conjunto de los verbos.

El principio de valencia que se asume en el presente planteamiento se caracteriza por explicar el número de complementos haciendo referencia al contenido semántico del predicado de la frase.

Procedemos a establecer la valencia del predicado de la frase y posteriormente la categoría de régimen, en la que se recoge la realización concreta de los complementos, para lo que hemos de recurrir a una frase concreta.

Para ello partimos - como ya indicamos - del significado del predicado, que para la frase (4) es sehen (ver). Sehen involucra dos objetos: el primero es el portador de la acción y el segundo el objeto de la acción. Asumimos, pues, un verbo bivalente. La categoría de régimen correspondiente es $\mathrm{NOM}+\mathrm{AKK}$, puesto que el primer complemento ha de ser una forma en nominativo y el segundo una forma en acusativo.

La estructura correspondiente a ( 4 ) es más compleja que la de ( 3 ) ya que une en un plano más inmediato las dos unidades sintácticas que juntas constituyen la forma verbal perifrástica. De ese nivel parte la constituyente que en un plano superior es unida con las constituyentes ich y ihn que juntas forman la constituyente 'frase' en un plano superior.

Veamos como se establecen la relación de concordancia y de régimen una vez que se disponga ya de la información en la estructura de marcación: 


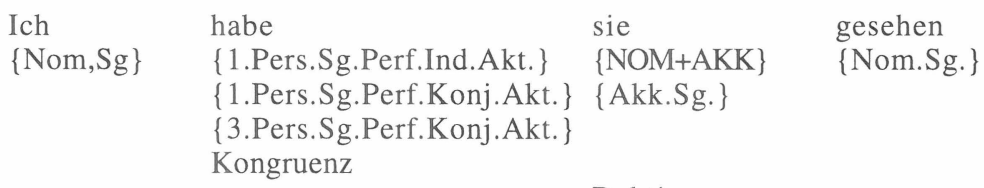

Rektion

De acuerdo con la categoría de régimen establecida en la categoría del paradigma del verbo sehen, ich cumple la condición de ser una forma en nominativo. Por consiguiente, desempeña la función sintáctica de sujeto con respecto al núcleo de la frase sehe. También sie reúne la condición de ser una forma en acusativo, por lo que se establece la relación de objeto de acusativo entre sie y sehe. El hecho de que sie sea también una forma en nominativo no tiene relevancia alguna. Como sie es la única forma marcada en 'acusativo', forzosamente tiene que ser el objeto en acusativo con respecto al predicado de la frase.

La relación tanto entre ich y sehen como entre sie y sehen es denominada 'régimen' (o "Rektion).

En cambio, entre ich y sehe existe además la relación de 'concordancia' (o "Kongruenz"), que se caracteriza por tener ambas constituyentes las mismas categorías en las categorías de unidad $\left(1^{\mathrm{a}}\right.$. persona singular). En este caso, efectivamente, resulta difícil determinar si es el verbo el que requiere determinadas formas, o al revés, si es el el sujeto de la frase, el que fija 'persona' y 'número'.

Por último tenemos el núcleo sehe que desempeña la función de predicado con respecto a la frase entera Ich sehe ihn:

el sujeto (f, s, e, S) $\quad=\{<i c h$, sehe $>\}$

el objeto de acusativo (f, s, e, S) $=\{<$ sie, sehe $>\}$

Para establecer la estructura de marcación resulta, efectivamente, mucho más fácil aplicar la Gramática Integrativa a una lengua como el alemán con sistema de casos, que p. ej. al español o al inglés, dado que no disponen de categorías como 'nominativo' o 'acusativo' que nos sirven de gran ayuda para averiguar el sujeto o el objeto.

Para la aplicación al español habría que realizar algunos cambios pertinentes en la estructura de marcación en la que se debería tener más en cuenta el orden de las constituyentes y la entonación, aspectos que todavía no han sido suficientemente estudiados.

A primera vista, este enfoque puede parecer algo complicado, pero permite tener en cuenta que dos unidades sintácticas formen una palabra léxica, pero también que dos palabras puedan desempeñar la misma función sintáctica. Este último caso puede ser relevante para el español

$$
\text { Le diré a Pablo, que venga luego. }
$$

donde le desempeña la misma función sintáctica que a Pablo.

En un plano inmediato se uniría le y a Pablo, dado que las dos palabras juntas forman el complemento directo. 
Este enfoque recoge varias realizaciones de los posibles complementos. Así, p. ej., el verbo alemán sehen no exige sólo una forma en acusativo, sino que admite además una oración completiva con $d a \beta$, una introducida por $o b \mathrm{y}$, por último, una pregunta indirecta ('indirekter Fragesatz' o ' $w$-Satz') $)^{7}$ :

$$
\begin{aligned}
& \text { Ich sehe, daß du Hunger hast. } \\
& \text { Yo veo que tu tienes hambre. } \\
& \text { Ich sehe, ob Otto vorbeikommt. } \\
& \text { Yo veo si pasa Otto. } \\
& \text { Ich sehe, wie du die Soße vorbereitest. } \\
& \text { Yo veo cómo preparas la salsa. }
\end{aligned}
$$

El verbo sehen exige dos complementos, un portador de la acción y lo que es objeto de tal acción, que, como vemos en las frases $(6-8)$, puede tratarse de una circunstancia ("Sachverhalt").

Esa circunstancia llena el lugar vacío igual que cualquier complemento, prueba de ello es que puede ser sustituido por el pronombre personal neutro es:

( 6a ) ( 7a ) ( 8a) Ich sehe es.

Obviamente se reflejaría en la Gramática Integrativa que la estructura es más compleja, pero seguirían siendo tres constituyentes las que se unirían bajo la categoría de constituyente 'frase' ('Satz'). Las frases ( 6 ) y ( 7 ) tienen la misma estructura, ( 8 ), en cambio, varía algo:

$$
\text { Ich sehe daß du Hunger hast }
$$

Ich sehe wie du die Soße zubereitest

La valencia es, pues, un principio significativo dentro de la Gramática Integrativa, pero sólo para determinar las funciones gramaticales. No afecta a la estructura de constituyentes en sí.

Recordemos que para diferenciar entre 'actantes' y 'complementos' Tesnière recurre a la categoría sintáctica:

Les actants son toujours des substantifs ou des équivalents de substantifs... Inversement les substantifs assument en principe toujours la fonction d' actants. (...) Les circonstants son toujours des adverbs (de temps, de lieu, de manière, etc.) ou des équivalents d'adverbes." 8

\footnotetext{
7 Algunos autores utilizan el término 'pregunta indirecta', como p. ej. P. Eisenberg, Grundriß der deutschen Grammatik (Stuttgart 1986) 331; G. Helbig "Probleme der Subklassifizierung der deutschen Nebensätze nach Form und Inhalt." Deutsch als Fremdsprache 4 (1982) 202.

Sin embargo, Engelen prefiere el término "W-Satz", Untersuchungen zu Satzbauplan und Wortfeld in der geschriebenen deutschen Sprache der Gegenwart. (München 1975) 97.

${ }^{8}$ L. Tesnière, Éléments de Syntaxe Structurale. (Paris 1980) 103.
} 
En un análisis más detallado podemos comprobar que no es tan fácil, sino que se hace necesario ampliar la teoría de modo que pueda tener en cuenta que también un sustantivo asume, en ocasiones, la función de circunstancial ("Bestimmung"):

$$
\begin{aligned}
& \text { Er schläft unter dem Baum. } \\
& \text { Él duerme debajo del arbol. }
\end{aligned}
$$

Pero también un adverbio puede desempeñar función completiva:

\section{Er wohnt hier.}

Él vive aquí.

Du bleibst dort.

Tu te quedas allí.

Un criterio para determinar si un sintagma es un complemento o un circunstancial es, como ya se ha visto, el referido a las propiedades lógico-semánticas del verbo. Si la frase sigue siendo gramatical omitiendo dicho sintagma, se trata de un constituyente constitutivo pero facultativo.

Sin embargo, si un sintagma no es exigido por propiedades lógico-semánticas, siempre será un circunstancial.

En la Gramática Integrativa, el hecho de que un sintagma desempeñe la función de circunstancial no repercute en la estructura de constituyentes. Tanto un complemento como un circunstancial es un constituyente de la frase. Es a la hora de establecer la función sintáctica de dicho constituyente cuando se recurre a una flecha para evidenciar entre qué constituyentes existe la relación gramatical en cuestión.

La razón de que este enfoque no permita discernir por la estructura de constituyentes complementos y circunstanciales es que tales distinciones son realizadas en un plano teórico que no se evidencian en absoluto en la estructura superficial. 